\title{
Lessons to be learned from an on-going Trust-wide audit of the malnutrition universal screening tool (MUST)
}

\author{
C. A. Vickery and D. N. Ramanaden \\ Nutrition Support Team, Conwy \& Denbighshire NHS Trust, Glan Clwyd Hospital, Bodelwyddan, \\ Denbighshire LL18 5UJ, UK
}

\begin{abstract}
A Trust-wide snapshot re-audit of compliance with MUST ${ }^{(1)}$ was undertaken in November 2006, 2.5 years after initial introduction of the tool into Conwy \& Denbighshire NHS Trust. All areas of the Trust were included with the exception of paediatrics, mental health and maternity. All areas had received initial training at time of introduction, consisting of either two or three sessions per ward area. Information was cascaded down to staff unable to attend. Further training on an on-going basis following previous audits (September 2004 and January 2006), and most recently training was delegated to individual dietitians covering their own ward areas during the 6 months before this latest audit. Their remit was to provide as much training as was deemed necessary in each area.

The aim was to assess compliance with the tool and compare with previous audits to establish areas of improvement and further training needs. Each ward area had an unscheduled visit and every patient present on the ward was audited using the established audit tool.

A total of $40 \%$ of patients had MUST completed in full (i.e. all parts of the form completed in full with action plan and evidence of action). This result compares with the 2004 audit showing 13\% completed in full. The average of Trust patients who had been given the correct MUST score was 34\%. (Of those screened, $51 \%$ had been given the correct score.) This average value was assessed by the author re-scoring each patient. Fifteen ward areas achieved correct scores of $>34 \%$, and seven wards achieved $>64 \%$. Of the fifteen ward areas not achieving the Trust average of $34 \%$ correct scores, seven areas scored $0 \%$.

The average level of compliance across the Trust does not reflect the good practice taking place on certain wards, e.g. certain wards achieved $70-100 \%$ compliance.

Following these results there is an obvious need for further intensive training to aid compliance. An action plan has been agreed with the Heads of Nursing. A minimum of three further training sessions is required, especially for areas with $<70 \%$ correct scores. This plan included: sessions to be recorded even if cancelled by the ward; names of nurses trained and grades to be recorded; training to begin with the most senior staff; go through individual audit results with each ward; include case studies in the training programme; ward managers to carry out their own interim audits; training only to be regarded as complete after discussion with ward manager; standardised training guidelines for dietitians to follow.
\end{abstract}

1. Malnutrition Advisory Group (2003) The MUST Report. Nutritional Screening of Adults: A Multidisciplinary Responsibility. Maidenhead, Berks.: BAPEN. 